\title{
An Improved Identification Method for Multivariable System
}

\author{
Qibing Jin, Kewen Wang*, Sajid Khursheed and Qi Wang \\ Institute of Automation, Beijing University of Chemical Technology, Beijing, China
}

Received: 6 Aug. 2013, Revised: 8 Nov. 2013, Accepted: 9 Nov. 2013

Published online: 1 Jul. 2014

\begin{abstract}
Due to many classical identification methods cannot be directly used for closed-loop control system, an improved identification method is proposed to simultaneously identify model parameters and the structure. The improved identification method used the genetic algorithm to estimate the initial search scope for the PSO algorithm, and then used the search result as the initial value of the Rosenbrock algorithm. On the basis the genetic algorithm to estimate is introduced to provide the rough initial search scope for the presented algorithm to improve the validity and accuracy. Simulation results show that compare with the PSO algorithm, the inertia weight variation PSO algorithm and the PSO-SQP algorithm proposed by Qibing Jin et al, the presented algorithm improves the optimizing efficiency of the particle swarm.
\end{abstract}

Keywords: initial neighborhood, PSO-R, closed loop identification, multivariable identification

\section{Introduction}

To obtain object dynamic mathematical model is the basis of advanced control. In order to gain an accurate process model, many researchers proposed different identification methods. But these methods depend largely on the collection data, but if working under strong noise disturbance and insufficiency response condition, it may generate a large estimate bias.

In the open loop condition, it only needs persistent excitation, the system can always be identified. But in industrial control, in consideration of the factors of stability, security and economy, open loop identification always not be allowed, so closed loop identification is necessary needed, especially in the system is unstable in open loop or the system contains feedback mechanisms condition [1,2]. Conventional identification methods such as Least Squares estimate method and Maximum Likelihood method [3]. These methods can obtain accuracy results in open loop system. However, when these methods directly apply to closed-loop system may generate large estimation deviation, even lead to unidentified. For closed loop system, searching method is a very effective identification method. Pan proposed the nonlinear stochastic (NLJ) based on LJ [4]. NLJ method improved the convergence speed of the search. But the identification results depend largely on the choice of initial parameters.

Particle swarm optimization (PSO) is an evolutionary computation technique developed by Dr. Eberhart and Dr. Kennedy in 1995 [5], inspired by social behavior of bird flocking. Compare with conventional identification methods, PSO method has many advantages such as simple computation, rapid convergence capability and without any requirement for the input and output data. PSO has been extended to many fields $[6,7,8]$. But, in practical application, PSO method has the limitations of converging to undesired local solution or premature convergence [9]. Later many improved PSO methods are proposed to solve these problems. Eberhart proposed a discrete binary PSO method which can limit dimensional position [10]. Shi proposed a linear decreasing weight PSO method which can improve the ability of local search by modifying weight value [11]. Clerc introduced constriction factor to improve the ability of local search [12]. These methods be restricted to change learning factors and weight values and cannot meet the requirements for identification accuracy in modern industrial control.

In this paper we present a improve method to estimate model parameter and the structure simultaneously. The method (Named PSO-R) combines the global search

\footnotetext{
*Corresponding author e-mail: heaven996@ hotmail.com
} 
capability of PSO and exactly local optimization of Rosenbrock. To improve the algorithm performance, we introduce genetic algorithm to estimate the rough search scope of PSO, this step can avoid the local search trap or premature convergence.Compare with the basic particle swarm optimization algorithm, Inertia Weight Variation PSO algorithm and PSO-SQP [13] proposed by Qibing Jin, Through simulation can be seen that the PSO-R method is successfully applied to multivariable system identification and achieves better results in the experiment.

\section{Preliminaries: the classic optimization algorithms - PSO and Rosenbrock}

\subsection{The main aspects of basic particle swarm optimization (PSO)}

The mathematical description of Particle Swarm Optimization is: within a $d$ dimension search place, each particle is looked as a node in place. Each particle depends on three vectors: a position vector $x_{i}=\left(x_{i, 1}, x_{i, 2}, \cdots, x_{i, d}\right)$ a velocity vector $v_{i}=\left(v_{i, 1}, v_{i, 2}, \cdots, v_{i, d}\right)$ and its experience vector $p_{i}=\left(p_{i, 1}, p_{i, 2}, \cdots, p_{i, d}\right)$. For each iteration, each particle according to the formulas below updated its speed and position:

$$
\begin{gathered}
v_{i, j}(t+1)=w v_{i, j}(t)+c_{1} r_{1}\left[p_{i, j}-x_{i, j}(t)\right] \\
+c_{2} r_{2}\left[p_{g, j}-x_{i, j}(t)\right] \\
x_{i, j}(t+1)=x_{i, j}(t)+v_{i, j}(t+1)
\end{gathered}
$$

where $v_{i, j}(t+1)$ and $x_{i, j}(t+1)$ are the current velocity and the current position, $v_{i, j}(t)$ and $x_{i, j}(t)$ are the previous velocity and the previous position. The learning factors $c_{1}$ and $c_{2}$ are set constant value, normally $c_{1}$ and $c_{2}$ are taken as $2, r_{1}$ and $r_{2}$ are random numbers between $[0,1], w$ is inertia weight which used to control the influence of previous velocity on the current velocity. The inertia weight $w$ is a very important parameter in PSO algorithm and could be used to control algorithm exploration ability and exploitation ability. The larger inertia weight $w$ is helpful to enhance the global search ability of algorithm and jump out the local optimum. In the later phase of the PSO algorithm, the smaller inertia weight is helpful to improve the local search ability and make the algorithm converge.

\subsection{The basic principle of Rosenbrock algorithm}

Rosenbrock algorithm is a method to solve unconstrained multiple optimization problem. The description of the problem can use the minimum value of object function as follow:

$$
\min f(x), x \in R^{n}
$$

The basic principle of Rosenbrock algorithm is: construct $n$ orthogonal vectors at the current position and then search in each direction, find the direction of the maximum function value decrease and move one step, reconstruct $n$ orthogonal vectors at the new position and repetitive operation above.

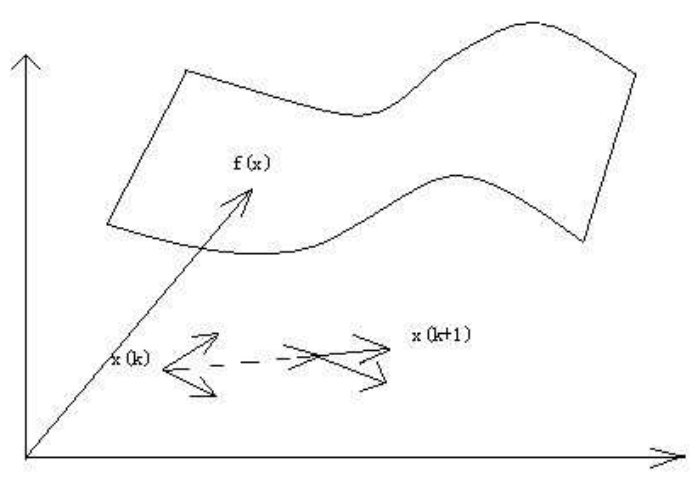

Fig. 1: The Rosenbrock method diagram

The algorithm is depicted as follow:

Step 1:Set initial estimated value $x^{(0)},\left\{d^{0}, d^{1}, \cdots, d^{n}\right\}$ are $n$ initial standard orthogonal vectors, the initial step length is $\delta^{0}=\left(\delta_{1}^{0}, \delta_{2}^{0}, \cdots, \delta_{n}^{0}\right)^{T}>0$, and set the parameters $\alpha, \beta$ and accuracy $\varepsilon>0$, set $k=0$;

Step 2:Make $y=x^{k}$

Step 3:Make $y$ as the basic point, move axially parallel to $\left\{d^{0}, d^{1}, \cdots, d^{n}\right\}$,

If $f\left(y+\delta_{j}^{k} d^{j}\right) \leq f(y)$, then

$$
y=y+\delta_{j}^{k} d^{j}, \delta_{j}^{k}=\alpha \delta_{j}^{k}
$$

$$
\begin{aligned}
& \text { If } y=y+\delta_{j}^{k} d^{j}, \delta_{j}^{k}=\alpha \delta_{j}^{k} \text {, then } \\
& y=y, \delta_{j}^{k}=-\beta \delta_{j}^{k}
\end{aligned}
$$

Until failures appear in $n$ directions;

Step 4:Set $x^{k+1}=y$, if $\left\|x^{k+1}-x^{k}\right\| \leq \varepsilon$, then stop the iteration, the output is $x^{k+1}$, or go to the next step;

Step 5:Set $\tilde{d}=x^{k+1}-x^{k}$, build new orthogonal vectors $\left\{d_{0}, d_{1}, \cdots, d_{n}\right\}_{k+1}$, set $\delta^{k+1}=\delta^{0}, k=k+1$, turn to step 3 .

According to the experience, $\alpha \in[2,3], \beta \approx 0.5$, in the initial stage orthogonal vectors can set as unit vectors. 


\section{PSO-R algorithm}

\subsection{Initial neighborhood}

Most optimization algorithms exist this problem: search algorithm itself has the randomness and will cause blind inefficient in optimization process. In this paper, we use the genetic algorithm to estimate the rough range search space of PSO algorithm, improves the convergence and accuracy of search process, avoid randomness factor of the PSO algorithm cause the local search trap or premature convergence.

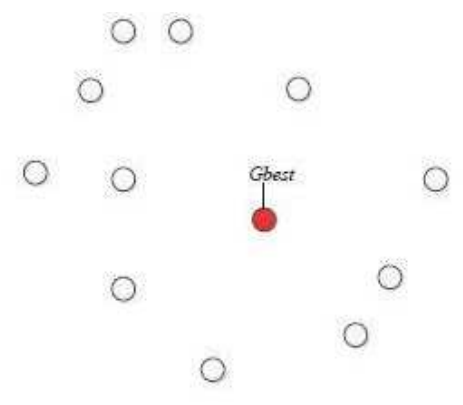

Fig. 2: Chart of Standard PSO algorithm searching process

Assume the red dot is the global optimum, the PSO algorithm random generate $N$ nodes in the whole search scope, and then according the formulas of PSO algorithm to calculate the optimum.

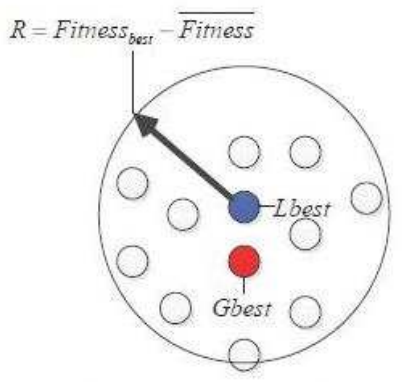

Fig. 3: Chart of Basing on the initial neighborhood of PSO algorithm optimization process

Assume the red dot is the global optimum, and the blue dot is the local optimum. The initial neighborhood in this paper, the center of initial value can be obtain by genetic algorithm. The radius $R$ of initial neighborhood is the difference of the optimal value and average optimal value of GA algorithm, this constitutes the initial neighborhood of PSO algorithm.

\subsection{PSO-R Algorithm}

Step 1:Using the GA algorithm to estimate the initial neighborhood of the PSO algorithm.

Step 2:Initialized parameters in PSO algorithm, including initial search position and particle speed.

Step 3:Comparison fitness value, if current fitness evaluation is better than the previous, let the current replace the previous.

Step 4:Update the velocity of each particle and the coordinate position.

Step 5:Judging the iteration number whether satisfy the set value, if satisfy according the formula 3 to update the position and speed of particle, and go to the next step, otherwise go to the step 2.

Step 6:Using the result of PSO algorithm as initial point of Rosenbrock, the results of the parameters estimation are $\hat{\theta}_{q}$, precision is $\varepsilon_{q}, q$ is the cycling time. According $J=\sum_{t=0}^{N}\left(y(t)-y_{e}(t)\right)^{2}$ to update the initial value of PSO and the cycling time.

$$
\hat{\theta}_{q+1}^{0}=\left(\hat{\theta}_{q+1}^{+}+\hat{\theta}_{q+1}^{-}\right) / 2
$$

where

$$
\begin{gathered}
\left\{\begin{array}{c}
\hat{\theta}_{q+1}^{+}=\hat{\theta}_{q}+\operatorname{abs}\left(\operatorname{rand}\left(\hat{\theta}_{q}-\hat{\theta}_{q-1}\right)\right) \\
\hat{\theta}_{q+1}^{-}=\hat{\theta}_{q}-\operatorname{abs}\left(\operatorname{rand}\left(\hat{\theta}_{q}-\hat{\theta}_{q-1}\right)\right)
\end{array}\right. \\
\varepsilon_{q+1}=\operatorname{rand} \times \varepsilon_{q}
\end{gathered}
$$

Step 7:Gain the global optimal value $\hat{\theta}_{k, l}$, and the corresponding optimal fitness value $f\left(\hat{\theta}_{k, l}\right)$, where the main iteration time in PSO algorithm is $k, l$ is the serial number of the particle in the group.

Step 8:Through calculate obtain the optimal value and the corresponding fitness function value. In turn down as $\hat{\theta}_{r}, f\left(\hat{\theta}_{r}\right)$, if $f(\hat{\theta})<f(\hat{\theta})$ let $\hat{\theta}=\hat{\theta}_{r}$.

Step 9:Along the $\hat{\theta}$ axis direction to search. Searching make fitness function minimum variable value, if $f\left(\hat{\theta}+v_{1} d_{j}\right) \leq f(\hat{\theta}), \hat{\theta}=\hat{\theta}+v_{1} d_{j}$.

Step 10:Determine whether meet the requirement ||$\theta_{k+1}-\theta_{k}|| \leq \varepsilon_{q}$, if satisfy, end the computation and output the optimal result $\theta_{k+1}$ or go to step 11 .

Step 11:Update orthogonal vector group for $n$ search directions, and use to set the initialization parameter forward $\sigma^{k+1}$ step, go to step 9. Figure 5 is the flow chart of the PSO-R algorithm.

\section{Simulation}

$$
\begin{aligned}
& \text { 4.1 First order plus dead time (FOPDT) model } \\
& G s=\frac{k_{p}}{\tau_{p} s+1} e^{-\theta s}
\end{aligned}
$$

Based on closed loop step test in terms of a P-type controller $(k c=0.5)$, with a step change of $h=0.05$ to 


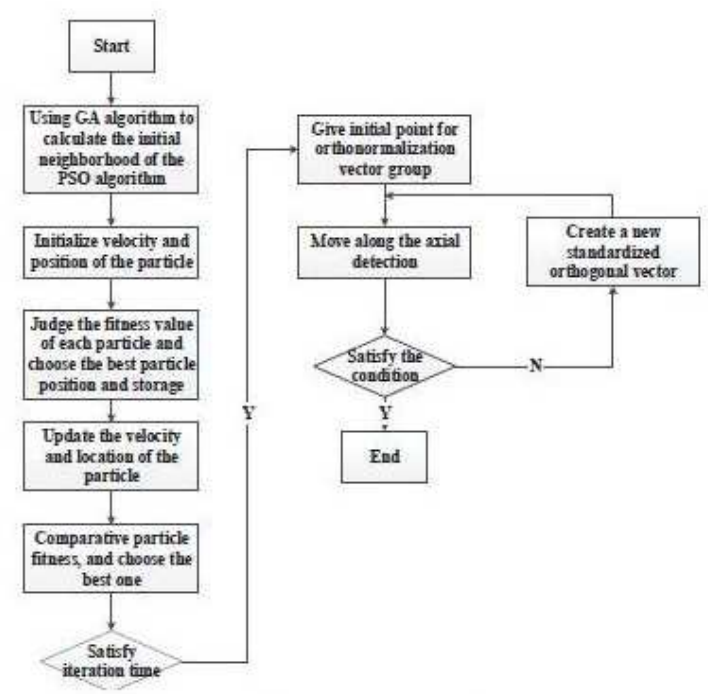

Fig. 4: Algorithm chart of PSO-R

the set point, we choose $G s=\frac{12.8}{16.7 s+1} e^{-s}$ as an example of FOPDT model. The basic PSO algorithm, the inertia weight variation PSO Algorithm, the PSO-SQP algorithm and the proposed algorithm are respectively used to estimate the selected model.

A Gaussian white noise respectively generating $N S R=5 \%, 10 \%$ and $20 \%$ is added to the output to test the robustness.

Table 1: The results of various algorithm for FOPTD model in no noise

\begin{tabular}{cccc}
\hline Actual Value & 12.8 & 16.7 & 1 \\
\hline PSO & 12.7989 & 16.6989 & -1.0022 \\
WPSO & 12.7999 & 16.6982 & -1.0091 \\
PSO-SQP & 12.8000 & 16.7000 & -0.9989 \\
PSO-R & 12.8000 & 16.7000 & -1.0000
\end{tabular}

Table 2: The results of various algorithm for FOPTD model in $N S R=5 \%$ noise

\begin{tabular}{cccc}
\hline Actual Value & 12.8 & 16.7 & 1 \\
\hline PSO & 12.7841 & 16.6916 & -0.9901 \\
WPSO & 12.8089 & 16.7110 & -0.9598 \\
PSO-SQP & 12.7998 & 16.6970 & -0.9972 \\
PSO-R & 12.8000 & 16.7007 & -1.0002 \\
\hline
\end{tabular}

Fig. 5 depicts the result of the PSO-R from step response test for FOPDT model. Table 1 to table 4 demonstrates that the search optimization method with frequency response to estimate the initials can obtain an excellent performance of FOPDT model identification in
Table 3: The results of various algorithm for FOPTD model in $N S R=10 \%$ noise

\begin{tabular}{cccc}
\hline Actual Value & 12.8 & 16.7 & 1 \\
\hline PSO & 12.7928 & 16.7140 & -0.8338 \\
WPSO & 12.8014 & 16.8495 & -1.0572 \\
PSO-SQP & 12.8008 & 16.7017 & -1.0012 \\
PSO-R & 12.8000 & 16.6987 & -0.9982 \\
\hline
\end{tabular}

Table 4: The results of various algorithm for FOPTD model in $N S R=20 \%$ noise

\begin{tabular}{cccc}
\hline Actual Value & 12.8 & 16.7 & 1 \\
\hline PSO & 12.8006 & 16.7435 & -0.8338 \\
WPSO & 12.8214 & 16.9926 & -1.1901 \\
PSO-SQP & 12.8214 & 16.6928 & -1.1901 \\
PSO-R & 12.8000 & 16.6999 & -1.0060 \\
\hline
\end{tabular}

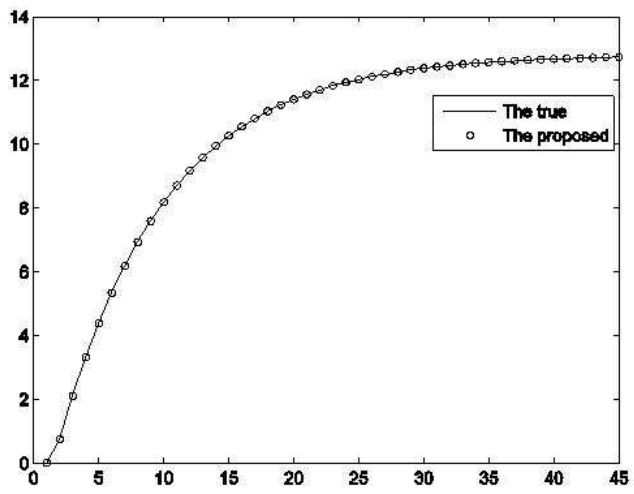

Fig. 5: Step responses test for FOPDT model estimated by PSO$\mathrm{R}$

spite of the existence of the noise and the complexity of the equivalent input; among the four algorithms, the proposed PSO-R achieves better results than PSO, WPSO and PSO-SQP in terms of accuracy and robustness.

\subsection{Second order plus dead time (SOPDT) model $G s=\frac{k_{p}}{t_{1} s^{2}+t_{2} s+1} e^{-\theta s}$}

A unit step is given to the SOPDT model of $G s=\frac{1}{12 s^{2}+8 s+1} e^{-s}$ in terms of a P-type controller $(k c=0.5)$, with a step change of $h=0.05$ to the set point. Experiments with the measurement noises of $N S R=5 \%$, $10 \%$ and $20 \%$ are respectively taken for PSO, WPSO, PSO-SQP and the proposed PSO-R with initial parameter estimate. The results are listed in Table 2.

The persistency of the excitation is a key issue for the identification of the system, whereas many methods need a persistent excitation during the whole process. In the 
Table 5: The results of various algorithm for SOPTD model in no noise

\begin{tabular}{cccc}
\hline Actual Value & 1 & 12 & 8 \\
\hline PSO & 1.0006 & 11.1810 & 7.9299 \\
WPSO & 0.9999 & 12.3526 & 8.0258 \\
PSO-SQP & 1.0000 & 12.1500 & 8.0049 \\
PSO-R & 1.0000 & 12.0080 & 8.0007 \\
\hline
\end{tabular}

Table 6: The results of various algorithm for SOPTD model in $N S R=5 \%$ noise

\begin{tabular}{cccc}
\hline Actual Value & 1 & 12 & 8 \\
\hline PSO & 1.0004 & 11.0869 & 7.9235 \\
WPSO & 1.0002 & 11.5059 & 7.9583 \\
PSO-SQP & 1.0000 & 11.7049 & 7.9933 \\
PSO-R & 1.0000 & 11.9868 & 7.9991 \\
\hline
\end{tabular}

Table 7: The results of various algorithm for SOPTD model in $N S R=10 \%$ noise

\begin{tabular}{cccc}
\hline Actual Value & 1 & 12 & 8 \\
\hline PSO & 1.0013 & 10.7953 & 7.9078 \\
WPSO & 1.0006 & 10.4549 & 7.8918 \\
PSO-SQP & 1.0004 & 11.0517 & 7.8997 \\
PSO-R & 1.0001 & 11.7738 & 7.9819 \\
\hline
\end{tabular}

Table 8: The results of various algorithm for SOPTD model in $N S R=20 \%$ noise

\begin{tabular}{cccc}
\hline Actual Value & 1 & 12 & 8 \\
\hline PSO & 1.0004 & 10.5602 & 7.8801 \\
WPSO & 1.0004 & 10.4046 & 7.8629 \\
PSO-SQP & 1.0002 & 11.0049 & 7.8777 \\
PSO-R & 1.0002 & 11.6753 & 7.9776 \\
\hline
\end{tabular}

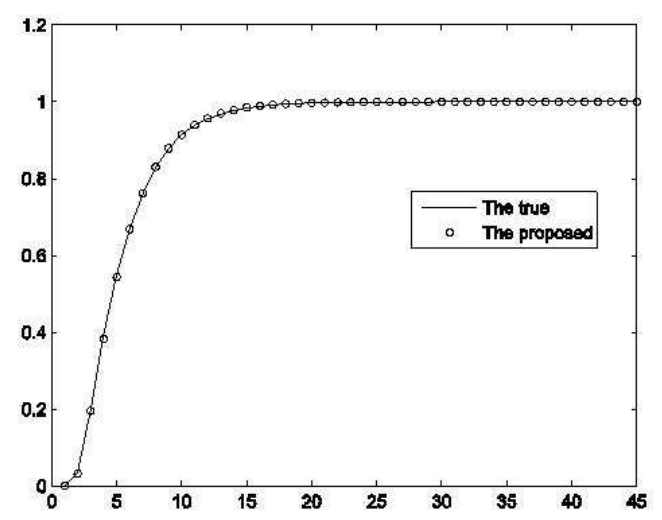

Fig. 6: Step responses test for SOPDT model estimated by PSO$\mathrm{R}$

paper, we respectively make the experiment with different excitation time and the results are as Fig. 7.
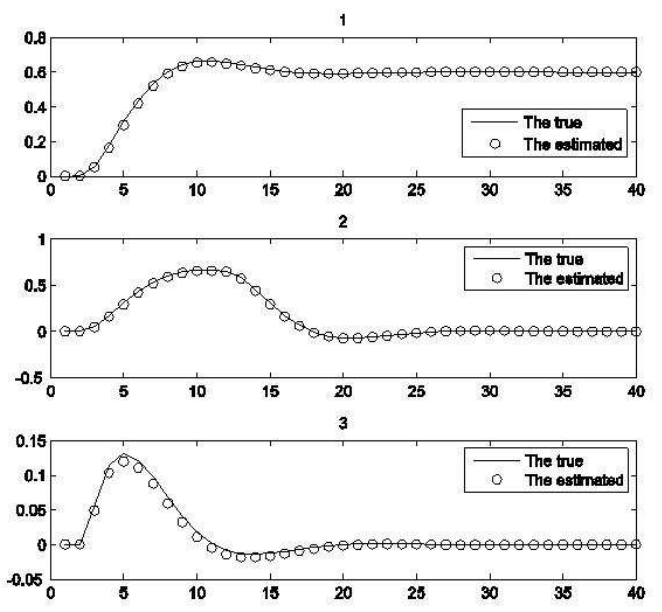

Fig. 7: The results of PSO-R with different excitation time

Subfigure 1 indicates a persistent excitation all time, Subfigure 2 depicts the excitation time $t=10 \mathrm{~s}$ and Subfigure 3 shows the excitation time $t=1 \mathrm{~s}$. The presented method is feasible for the non-sustainable excitation whereas the stability and accuracy of the results may be affected at a certain degree, hence a persistent excitation is preferable in the simulations of SISO system.

\subsection{Woodberry model}

Woodberry model is proposed by Wood and Berry in 1973 and widely adopted in the identification of multivariable system because of its characteristics of strong coupling and multiple time delays between the various loops. We get a good result with PSO-R in the experiment, have also made very good identification results.

Woodberry model

$$
G(s)=\left[\begin{array}{cc}
\frac{12.8 e^{-s}}{1+16.7 s} & \frac{-18.9 e^{-3 s}}{1+21 s} \\
\frac{6.6 e^{-7 s}}{1+10.9 s} & \frac{-19.4 e^{-3 s}}{1+14.4 s}
\end{array}\right]
$$

For the decentralized closed loop system with $k c 1=$ $0.5, k c 2=-0.09$, the step tests are respectively taken by $r 1=1, r 2=2$ and $r 1=4, r 2=2$. Choose $G_{c i}\left(r_{i}-y_{i}\right)$ as the equivalent inputs in the simulation and results of PSO ,WPSO, PSO-SQP and the proposed method are listed in Table 9 to table 12.

\subsection{Multivariable model}

The transfer function of system being identified is

$$
y=1+x_{1}-1.5 x_{2}+1.6 e^{-x_{3}}+2 \sin \left(1+x_{4}\right)
$$


Table 9: The results estimated channel $G 11$ by various algorithms

\begin{tabular}{cccc}
\hline Actual Value & 12.8 & 16.7 & -1 \\
\hline PSO & 12.7929 & 15.6 & -1.21 \\
WPSO & 12.7949 & 16.6193 & -1.0044 \\
PSO-SQP & 12.7984 & 16.3140 & -1.0027 \\
PSO-R & 12.8000 & 16.6942 & -1.0014 \\
\hline
\end{tabular}

Table 10: The results estimated channel $G 12$ by various algorithms

\begin{tabular}{cccc}
\hline Actual Value & -18.9 & 21 & -3 \\
\hline PSO & -18.7700 & 18.2000 & -2.8500 \\
WPSO & -18.8419 & 20.2100 & -2.93 \\
PSO-SQP & -18.7280 & 20.9420 & -3.0017 \\
PSO-R & -18.9000 & 21.0000 & -3.0002 \\
\hline
\end{tabular}

Table 11: The results estimated channel $G 21$ by various algorithms

\begin{tabular}{cccc}
\hline Actual Value & 6.6 & 10.9 & -7 \\
\hline PSO & 6.5656 & 10.9218 & -6.5500 \\
WPSO & 6.5766 & 10.9109 & -6.7630 \\
PSO-SQP & 6.5856 & 10.9081 & -6.9852 \\
PSO-R & 6.6000 & 10.9000 & -6.9975 \\
\hline
\end{tabular}

Table 12: The results estimated channel $G 22$ by various algorithms

\begin{tabular}{cccc}
\hline Actual Value & -19.4 & 14.4 & -3 \\
\hline PSO & -19.8800 & 13.2700 & -2.4000 \\
WPSO & -19.3074 & 14.4339 & -2.7300 \\
PSO-SQP & -19.3942 & 14.4140 & -3.0060 \\
PSO-R & -19.4000 & 14.4001 & -3.0007 \\
\hline
\end{tabular}
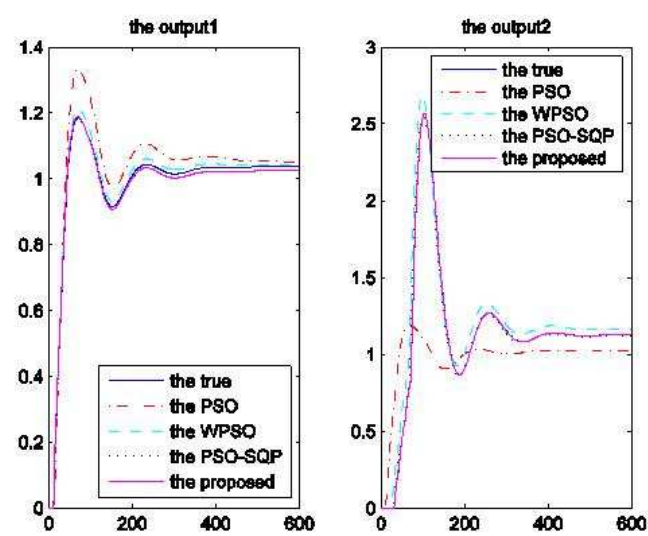

Fig. 8: The results of the various algorithm for Woodberry model

According to formula 8 generate 30 groups sample model. Assumed we didn't know the structure of model in advanced, chosen all of the meta-model to identify the system. Set the parameters of BPSO algorithm in identification process as follow: particle number $N=20$, inertia weight $w=1$, accelerate factors $C_{1}=2, C_{2}=1.65$, $X_{\max }=2$, the max evolution number is 1200 . Set inertia weight in WPSO algorithm as $X_{\max }=0.9, X_{\min }=0.4$. Other parameters are the same as BPSO algorithm.

After many times simulation, chosen a better group result as follow:

PSO algorithm is

$$
\begin{aligned}
y= & 0.2584+1.4775 x_{1}-1.9802 x_{2}+1.9991 e^{-1.3560 x_{3}} \\
& +2.1390 \sin \left(1.9882+1.0138 x_{4}\right.
\end{aligned}
$$

WPSO algorithm is

$$
\begin{aligned}
y= & 0.3793+1.0683 x_{1}-1.5760 x_{2}+1.2469 e^{-0.8776 x_{3}} \\
& +2.0376 \sin \left(0.9791+1.0013 x_{4}\right)
\end{aligned}
$$

PSO-SQP algorithm is

$$
\begin{aligned}
y= & 0.9970+0.9956 x_{1}-1.5042 x_{2}+1.5896 e^{-0.9734 x_{3}} \\
& +2.0027 \sin \left(0.9988+1.0001 x_{4}\right)
\end{aligned}
$$

PSO-R algorithm is

$$
\begin{aligned}
y= & 0.9984+0.9997 x_{1}-1.5000 x_{2}+1.5963 e^{-0.9734 x_{3}} \\
& +2.0000 \sin \left(1.0000+1.0000 x_{4}\right)
\end{aligned}
$$

Identification parameters of each algorithm are shown in table 13 .

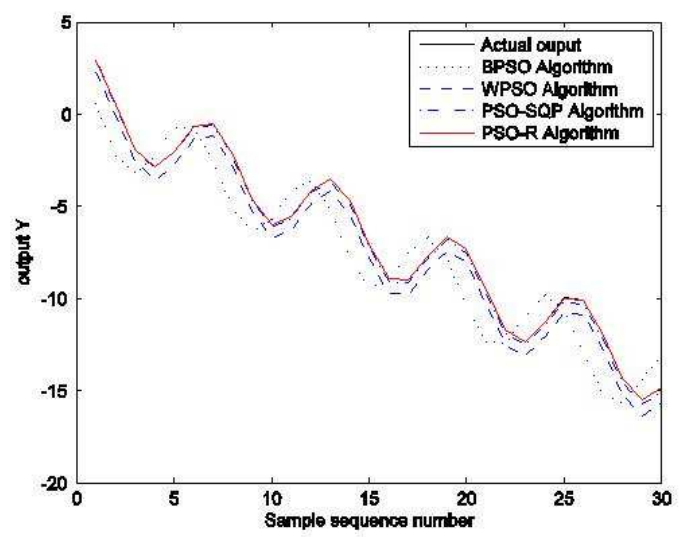

Fig. 9: Without noise cases of various algorithm system output curve

From the contrasts above we could see, the hybrid algorithm combined particle swarm optimization algorithm and sequential quadratic programming algorithm identify system output curve is more close to the real value, precision obviously improved. 
Table 13: Various algorithm system parameter identification results

\begin{tabular}{ccccccccc}
\hline $\begin{array}{c}\text { Actual } \\
\text { Value }\end{array}$ & 1 & 1 & -1.5 & 1.6 & -1 & 2 & 1 & 1 \\
\hline BPSO & 0.2584 & 1.4775 & -1.9802 & 1.9991 & -1.3560 & 2.1390 & 1.9882 & 1.0138 \\
WPSO & 0.3793 & 1.0683 & -1.5760 & 1.2469 & -0.8776 & 2.0376 & 0.9791 & 1.0013 \\
PSO-SQP & 0.9970 & 0.9956 & -1.5042 & 1.5896 & -0.9734 & 2.0027 & 0.9988 & 1.0001 \\
PSO-R & 0.9984 & 0.9997 & -1.5000 & 1.5963 & -0.9897 & 2.0000 & 1.0000 & 1.0000 \\
\hline
\end{tabular}

\section{Conclusion}

In this paper, we present an improved identification method for multivariable system. The idea is to search the local optimization with Rosenbrock algorithm at the set iteration of PSO and get a better result as the best global location. With the rough search scope of PSO algorithm estimated by the genetic algorithm, the search optimization approaches are improved in convergence speed and robustness, experiment is utilized to estimate the FOPDT and SOPDT under the disturbances of different NSR use PSO, WPSO, PSO-SQP and PSO-R are respectively.The results of simulation prove that PSO-R with the advantage of the global search capability of particle swarm optimization (PSO) algorithm and exactly local optimization of Rosenbrock algorithm, is an approximate unbiased and effective identification method that can be successfully applied to the model of the closed loop identification with large noise, time delay. Finally, we also use PSO-R method to identify multivariable closed loop system, compared with other methods the method proposed by this article get a better result as well.

\section{Acknowledgement}

This work is partially supported by the financial support of the National High-Tech Research and Development Plan of China (Grant 2008AA042131) and the National Grand Fundamental Research 973 Program of China (Grant 2007CB714300) and partly by the Automation Institute Beijing University of Chemical Technology. The authors are grateful to the anonymous reviewers for their valuable recommendations.

\section{References}

[1] L. Ljung, System Identification: Theory for the User, Prentice-Hall, Englewood Cliffs, NJ, (1999).

[2] Q. G. Wang, Y. Zhang, X. Guo, Robust closed-loop identification with application to auto-tuning, Journal of Process Control, 11, 519-530 (2011).

[3] R. Diversi, R. Guidorzi, U. Soverini, Maximum likelihood identification of noisy-input-output models, Automatica, 4, 464-472 (2007).

[4] L. D. Pan, The application of optimization for regulator selftuning online, Journal of Beijing University of Chemical Technology, 11, 17-18 (1984).
[5] J. Kennedy, R. C. Eberhart, Particle swarm optimization, in: International Conference on Neural Networks, Perth, Australia, (1995).

[6] D. B. Chen, C. X. Zhao, Particle swarm optimization with adaptive population size and its application, Applied Soft Computing, 9, 39-48 (2009).

[7] G. Quaranta, G. Monti, G. Carlo Marano, Parameters identification of Van der Pol-Duffing oscillators via particle swarm optimization and differential evolution, Mechanical Systems and Signal Processing, 24, 2076-2095, (2010).

[8] Y. H. Shi, R. C. Eberhart, A modified particle swarm optimizer, in: IEEE International Conference of Evolutionary Computation, AK, USA, (1998).

[9] P.K. Triapthi, S. Bandyopadhyay, S.K. Pal, Multi-objective Particle Swarm Optimization with time variant inertia and acceleration coefficients, Information Sciences, 177, 50335049 (2007).

[10] Kennedy J, Eberhart R C. A discrete binary version of the particle swarm algorithm, Proceeding of the World Multi-conference on Systemic, Cybernetics and Informatics. Piscataway, NJ: IEEE Service Center, 4104-4109 (1997).

[11] Shi Y, Eberhart R C, A modified particle swarm optimizer, IEEE International Conference of Evolutionary Computation, Anchorage, Alaska, (1998).

[12] Clerc M, The swarm and the queen: Towards a deterministic and adaptive particle swarm optimization, Proceedings of the Congress of Evolutionary Computation, Ishington, 19511957 (1999).

[13] Q. B. Jin, J. Zhang, L. Quan, L. T. Cao, Hybrid PSO-SQP for simultaneous identification of structure and parameters in a multivariate system, Control and Decision, 26, 1-5 (2011).

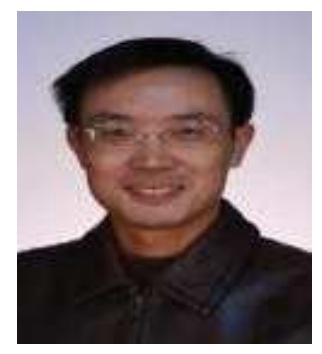

Qibing Jin received the MS degree from Northeast Petroleum University in 1996, and the $\mathrm{PhD}$ degree from the department of Optics Engineering, Northeastern University in 1999. He is currently a professor in Beijing University of Chemical Technology. His research interests are in the areas of advanced control and application in industry, process modeling and optimization, and multivariate prediction and internal model control. 


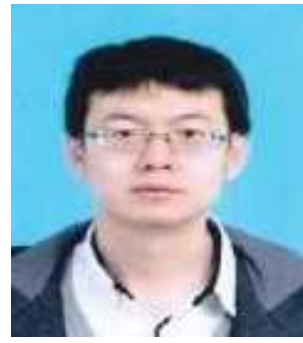

modeling and optimization and system identification. the MS degree from Liaoning Normal University in 2010, and the Phd degree from Institute of Automation in Beijing University of Chemical Technology in 2013. His research interests are in the areas of real-time database application, process

\section{Sajid Khursheed}

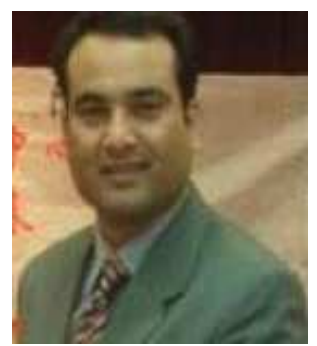
received the Phd degree from Institute of Automation in Beijing University of Chemical Technology in 2013. His research interest covers signal processing and denoising.

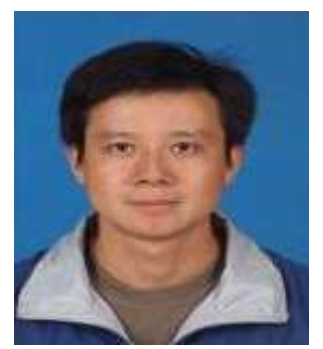

Qi Wang Lecturer at Beijing University of Chemical Technology and $\mathrm{Ph}$. D. candidate at the College of Information Science and Technology, Beijing University of Chemical Technology. His research interests are in the areas of advanced control and application in industry and multivariate prediction and internal model control. 